\title{
Evaluation of Results of Extraperiosteal Plating of Pronation-Abduction Ankle Fractures in Adults
}

\begin{abstract}
Tafhim Ehsan Kabir ${ }^{*}$
Salehuddin Ahmad ${ }^{2}$

Alak Kanti Biswas ${ }^{1}$
\end{abstract}

'Department of Orthopaedic Surgery Chattogram Maa-O-Shishu Hospital Medical College Chattogram, Bangladesh.

${ }^{2}$ Department of Orthopaedic Surgery Chattogram Medical College Hospital Chattogram, Bangladesh.
*Correspondence to:

Dr. Tafhim Ehsan Kabir

Assistant Professor

Department of Orthopaedic Surgery

Chattogram Maa-O-Shishu Hospital Medical College

Chattogram, Bangladesh.

Mobile : +8801712276487

Email : tafhimekf@yahoo.com

Date of Submission : $\quad 15.06 .2020$

Date of Acceptance ： 28.08 .2020

www.banglajol.info/index.php/CMOSHMCJ

\begin{abstract}
Background: Pronation-abduction ankle fractures frequently are associated with substantial lateral comminution and is associated with the highest rates of nonunion among indirect ankle fractures. It is one of the common injuries occurring in adult age group where trauma is the main etiology. The present prospective hospital based observational study was conducted to evaluate the functional outcome of treatment of ankle fracture by extraperiosteal plating in a series of patients with pronation-abduction ankle fractures.
\end{abstract}

Materials and methods : A total of 30 consecutive patients aged over 18 years with pronation-abduction ankle fracture were included in the study and underwent surgery by extraperiosteal plating of the fibular fracture. Patients were selected irrespective of sex. The average age of the patients was 36.5 years. Patients with gustilo type III open fractures, pathological fractures were excluded from the study. The patients were evaluated functionally with the use of the American Orthopaedic Foot and Ankle Society score (AOFAS) radiographically, and clinically with range of motion testing.

Results: Immediate postoperative and final follow-up radiographs showed that most of the patients had a well-aligned ankle mortise on the fractured side as compared with the normal side on the basis of standardized measurements. All fractures healed without displacement. The average American Orthopaedic Foot and Ankle Society score was 81.5 . The range of motion averaged $13^{\circ}$ of dorsiflexion and $53^{\circ}$ of plantar flexion. Satisfactory results were obtained in $93.33 \%$ patients. Complication includes 2(6.66\%) superficial infection, 1(3.33\%) deep infection, 4(13.33\%) delayed union, 1(3.33\%) skin necrosis. Radiological evaluation revealed persistent of talar shift in 1(3.33\%) patient postoperatively.

Conclusions: Extraperiosteal plating to be an effective method for the stabilization of pronation-abduction ankle fractures. The technique allows for accurate reduction of the mortise without stripping the periosteum of the comminuted region of the fracture. The technique is easier and faster than standard techniques in which the lateral periosteum is split to facilitate placement of the plate.

Key words : Pronation-Abduction ankle fractures; Extraperiosteal plating; Reduction.

\section{INTRODUCTION}

Ankle fractures are one of the most common fracture and one of the common injuries treated by orthopaedic surgeons and the ankle joint represents the most commonly injured weight-bearing joint. Approximately one in seven ankle fracture is accompanied by a distal tibiofibular syndesmotic disruption ${ }^{1,2}$. Most are lowenergy fractures of one or both malleoli, usually caused by a twisting mechanism resulting immediate pain, swelling and difficulties weightbearing . 
Other causes of ankle fracture are trauma (Especially motor vehicle accidents) sports injuries (i.e Football players) and osteoporosis in the elderly (Including postmenopausal women $)^{3}$.

Bimalleolar fractures have been the subject of numerous studies and articles regarding the mechanism of injury, classification and treatment modalities. The anatomy of the joint makes it very unstable in cases of fractures or ligamentous injuries of the ankle ${ }^{4}$. In the operative treatment of displaced ankle fracture anatomical reduction especially of the lateral malleolus is essential. Even a minimal displacement of the lateral malleolus alters the tibiotalar weight bearing surface possibly causing early arthrosis of the ankle joint ${ }^{5}$.

Despite extensive research identification of the mechanism of ankle fracture and the correlation of this information with clinical radiograph remain problematic. Knowing the precise mechanism of ankle fracture is important because it helps to assess the extent of soft tissue injury and the sequence of injury on the basis of the fracture pattern seen on radiograph ${ }^{6}$.

Injuries around the ankle joint causes destruction of not only the bony architecture, but also often the ligamentous and soft tissue component ${ }^{7}$.

Uunderstanding of ankle hindfoot motion is important for both evaluation and treatment of ankle disorders. Under the LaugeHansen classification system the two factors determining ankle fracture pattern are the position of the foot at the time of the traumatic events- supination or pronation and the direction of the deforming force abduction, adduction or external rotation ${ }^{8}$.

The most widely accepted current classification of ankle fractures are Lauge-Hansen and the AO classification. Both these classification systems describe a fracture pattern involving a fibula fracture above the inferior tibiofibular syndesmosis, in conjunction with a medial malleolus avulsion or a medial ligamentous rupture (Lauge-Hansen pronationabduction and AO type $\mathrm{C}$ ). These classification of ankle fractures describe the mechanism of injury as relative movement between the foot and the leg, defining which forces to counter in order to reduce and surgically stabilize a fracture 9 .

The deforming force in pronation-abduction fractures is translational rather than rotational, with the medial structures failing first, under tension, and the fibula fracturing last. This typically results in a transverse fracture line approximately 5 $\mathrm{cm}$ proximal to the joint with lateral comminution, consistent with bending failure.

Lateral comminution of the fibula makes the operative treatment of this type of ankle fracture challenging. Traditional subperiosteal plating techniques strip the remaining soft tissue from the fracture, making the fragments extremely difficult to align and stabilize with a lateral plate, which has led to recommend bone grafting for comminuted fractures. Extraperiosteal fixation techniques, which preserve the periosteum and indirectly reduce comminution, have been used for the treatment of other long bones. With the use of an extraperiosteal approach, the soft-tissue attachments to the fracture fragments are preserved, which maintains blood flow to promote healing and aids in obtaining the reduction ${ }^{10,11}$. Other methods of conventional treatments are conservative treatment using plaster cast immobilization, subperiosteal plating, pinning etc.

A thorough understanding of the ankle anatomy, mechanism of the injury, interpretation of the radiographs and adherence to basic principles of fracture management are the basis for a good result. Considering all of the above, it was tried to analyse the results of Pronation-abduction ankle fractures treated at Chittagong Medical College Hospital.

\section{MATERIALS AND METHODS}

This is an observational study was done in Orthopaedic Surgery Department of Chittagong Medical College Hospital from January 2012 to December 2013. Patients were selected irrespective of sex. For diagnosis purpose Lauge- Hansen classification of ankle fracture was used. All the patients were within 18 to 57 years of age with closed and type I and type II open fractures were selected for the study.

Patients with below 18 years of age were excluded from the study. Patients having uncontrol DM, CRF, COPD or medically unfit were excluded. Compound fracture eg. Gustilo type III, associated fracture arround ankle joints, more then 3 weeks old injuries and those who were not willing for surgery were excluded too from the study.

Total 34 patients were enrolled in the study, 4 patients were lost to follow up. So finally 30 patients were availabe for evaluation.

\section{RESULTS}

Table I : Distribution of patients according to dorsiflexion movement $(\mathrm{n}=30)$.

\begin{tabular}{lcc} 
Dorsiflexion & Frequency & Percentage \\
$0-14$ & 2 & $6.66 \%$ \\
$0-12$ & 26 & $86.68 \%$ \\
$0-10$ & 2 & $6.66 \%$ \\
\hline
\end{tabular}

In $2(6.66 \%)$ patient dorsi flexion range from $0-14^{0}, 26(86.68 \%)$ patients movement range from $0-12^{0}, 2(6.66 \%)$ patients it was $0-10^{0}$. Mean dorsiflexion range was $13^{\circ}$.

Table II : Distribution of patients according to planter flexion movement $(\mathrm{n}=30)$.

\begin{tabular}{lcc} 
Planter flexion & Frequency & Percentage \\
$0-54$ & 4 & $13.33 \%$ \\
$0-52$ & 23 & $76.67 \%$ \\
$0-50$ & 3 & $10 \%$ \\
\hline
\end{tabular}


In $4(13.33 \%)$ patient planter flexion range from $0-54^{0}$, $23(76.67 \%)$ patients movement range from $0-52^{0}, 3(10 \%)$ patients it was $0-50^{\circ}$. Mean planter flexion range was $53^{\circ}$.

Table III: Distribution of patients according to walking distances in block $(\mathrm{n}=30)$.

\begin{tabular}{lcc} 
Walking distances in block & Frequency & Percentage \\
$>6$ & 5 & $16.66 \%$ \\
$4-5$ & 13 & $43.35 \%$ \\
$2-3$ & 8 & $26.66 \%$ \\
$<1$ & 4 & $13.33 \%$ \\
\hline
\end{tabular}

In the study $5(16.66 \%)$ patients could cross 6 blocks by walking, $13(43.35 \%)$ cross 4 to 5 blocks, $8(26.66 \%)$ cross 2 to 3 blocks and $4(13.33 \%)$ could able to cross only 1 or less blocks.

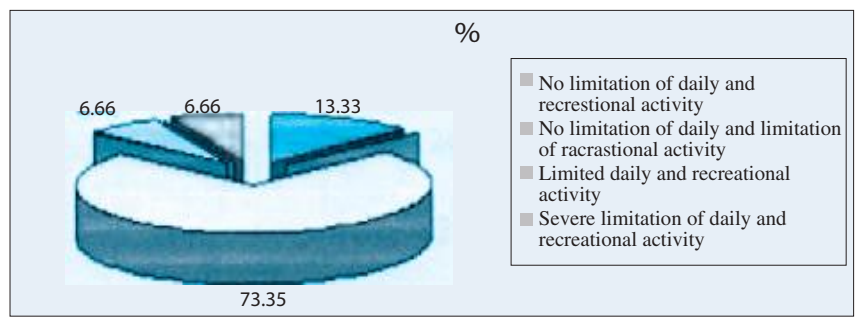

Figure 1 : Distribution of patients according to functional activities.

During follow up 4(13.33\%) patients had no limitation on activities, 22(73.35\%) had no limitation on daily but had limitation on recreational activities, $2(6.66 \%)$ had limited activities and $2(6.66 \%)$ had severe limitation on daily and recreational activities.

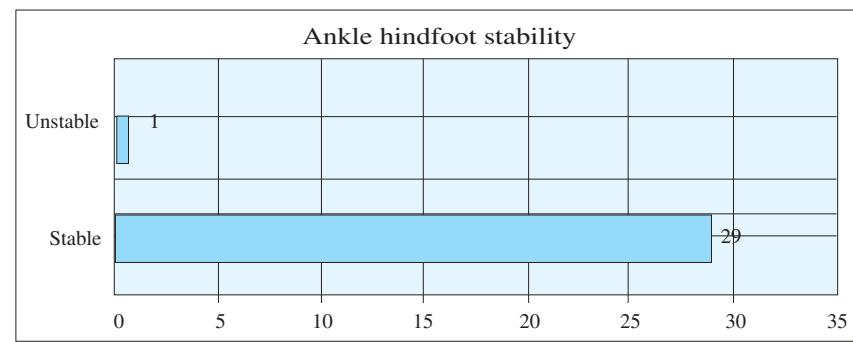

Figure 2 : Distribution of patients according to ankle hind foot stability.

In the study $29(96.67 \%)$ patients had stable ankle hind foot and only $1(3.33 \%)$ patient had unstable ankle hind foot stability.

Table IV: Distribution of patients according to AOFAS scoring system $(n=30)$.

\begin{tabular}{lcc} 
AOFAS scoring grading & Frequency & Percentage \\
$91-100$ & 0 & $0 \%$ \\
$81-90$ & 24 & $80 \%$ \\
$71-80$ & 2 & $6.66 \%$ \\
$61-70$ & 2 & $6.68 \%$ \\
$51-60$ & 2 & $6.66 \%$ \\
\hline
\end{tabular}

Out of 30 patients $24(80 \%)$ had score between 81 to 90 , $2(6.66 \%)$ patients had score within $71-80,2(6.68 \%)$ had score within 61-70 and 2(6.66\%) had score within 51-60. None of the patients had score between 91-100. Mean score was 81.5.

Table $\mathbf{V}$ : Distribution of patients according to the evidence of radiological healing.

$\begin{array}{lcc}\text { Radiological healing time } & \text { Frequency } & \text { Percentage } \\ 12 \text { week } & 6 & 20 \% \\ 14 \text { week } & 17 & 56.67 \% \\ 16 \text { week } & 3 & 10 \% \\ 18 \text { week } & 4 & 13.33 \%\end{array}$

In the study $6(20 \%)$ patients had radiological evidence of healing at 12 weeks, $17(56.67 \%)$ had healing evidence at 14 weeks. In 3(10\%) patients it was at 16 weeks, and in 4(13.33\%) patients the radiological evidence of healing was at or more than 18 weeks. Mean week of appearing radiological healing was 15 week.

Table VI : Evaluation of outcome at final follow up $(n=30)$.

\begin{tabular}{lcc} 
Final outcome & Frequency & Percentage \\
Satisfactory & 28 & $93.34 \%$ \\
Unsatisfactory & 2 & $6.66 \%$ \\
\hline
\end{tabular}

To determine the final outcome of the study the excellent and good grades according to AOFAS ankle-hind foot score was treated as satisfactory group and the poor grade was treated as unsatisfactory group. So a total of $28(93.34 \%)$ patients were in the satisfactory group and only $2(6.66 \%)$ patients were in unsatisfactory group.

\section{DISCUSSION}

Ankle fracture specially pronation abduction injury is not an uncommon problem encountered in the accident and emergency department of a hospital. However, there is a trend towards treating most of the fractures conservatively hoping that nature would do best. Unstable pronation-abduction ankle fractures result in laterally comminuted fibular shaft fractures because of the bending moment at the time of fracture. The resultant fragmented bone is challenging to adequately align while maintaining satisfactory vascularity for fracture-healing ${ }^{1}$.

A classic direct open fracture reduction and rigid plate fixation will violate the soft-tissue attachments of the fragments and jeopardize the viability of the injured bone. The surgical site is therefore placed at risk for nonunion and infection ${ }^{1,2}$. In this study $2(6.66 \%)$ patient dorsiflexion range from $0-14^{0}$, $26(86.68 \%)$ patients movement range from $0-12^{0}, 2(6.66 \%)$ patients it was $0-10^{\circ}$. Mean dorsiflexion range was $13^{\circ}$. Present series also shows that $4(13.33 \%)$ patient planterflexion range from $0-54^{0}, 23(76.67 \%)$ patients movement range from $0-52^{0}$, $3(10 \%)$ patients it was $0-50^{\circ}$. Mean planter flexion range was $53^{0}$. So the range of movement of present series corresponds 
with other series of Paul Tornetta III and Jodi Siegel ${ }^{2}$. The extraperiosteal position of the plate allows minimum soft tissue damage and provides the most biologically friendly environment for union. Present study showed 5(16.66\%) patients could cross 6 blocks by walking, $13(43.35 \%)$ cross 4 to 5 blocks, $8(26.66 \%)$ cross 2 to 3 blocks and $4(13.33 \%)$ could able to cross only 1 or less blocks. In a series of Brad Weening et al showed in their study that $18.5 \%$ and $4.2 \%$ of the patients developed post operative pain and stiffness. So the reduce ability to walk was due to postoperative pain and stiffness ${ }^{12}$.

During follow up 4(13.33\%) patients had no limitation on any activities, 22(73.35\%) had no limitation on daily but had limitation on recreational activities, $2(6.66 \%)$ had limited and $2(6.66 \%)$ had severe limitation on daily and recreational activities. Paul Tornetta III showed in a review study that $90 \%$ of the patients went to unlimited functional work and out of them $82 \%$ of patients went to normal daily and recreational activities $^{2}$. Using the extraperiosteal plate as a reduction tool allows the soft tissue envelop to be preserved and maintain appropriate length and alignment. All the fracture in the current series had a well aligned mortice too.

In this study $25(83.34 \%)$ patients developed mild, 3(10\%) patients developed moderate and 2(6.66\%) patients developed marked restriction on hind foot motions. Current study also showed that 29(96.67\%) patients had stable ankle hind foot and only $1(3.33 \%)$ patient had unstable ankle hind foot stability which is similar to the previous study of Jodi Siegel and Paul Tornetta III $^{2}$. A lateral buttress plate when usually applied outside of the periosteum bridges the comminuted fragments, an indirect reduction technique used for high fibula fracture associated with syndesmotic injury.

At follow ups in this series, each patient was assessed for pain on full weight bearing, functional activity, walking distance, gait abnormality, range of motion of ankle joints, ankle-hindfoot stability \& limitation of the activates of daily living and range of motion according to AOFAS score. In the present series out of 30 patients $24(80 \%)$ had AOFAS score between 81 to 90 which is regarded as excellent, $2(6.68 \%)$ patients had having score within 71-80 which is regarded as good, $2(6.66 \%)$ had score within $61-70$ which is fair and only $2(6.66 \%)$ have score in between 51 to 60 which is poor. None of the patients had score within 91-100. Mean score was 81.5. Paul Tornetta III and Jodi Siegel showed in their study the average AOFAS score of the patient at the lattest follow up was 82 (Range 68 to 100). N. Hamid et al showed in their study that the AOFAS score for the whole population was 85.2 (Range 55 to 100 ) and the mean score was 83.07 for group I, 92.4 for group II and 83.07 for group III respectively ${ }^{8}$. Extraperiosteal plating is an effective method for stabilization of pronation-abduction type of ankle fracturel. This technique allows for accurate reduction of the mortise without stripping the periosteum of the comminuted region of the fracture.
In the current study $6(20 \%)$ patients had radiological evidence of healing at 12 weeks, $17(56.67 \%)$ had healing evidence at 14 weeks. In $3(10 \%)$ patients it was at 16 weeks and in $4(13.33 \%)$ patients the radiological evidence of healing was at or more than 18 weeks. Mean week of appearing radiological healing was 15 week. Paul Tornetta III and Jodi Siegel showed in their study that the fibular fractures were healed without displacement at arround 10 weeks ${ }^{2}$. The excellent healing in time probably augmented due to extraperiosteal placing of plate. The endosteal blood supply is interrupted in association with comminuted shaft fracture and the vitality of the bone depends on periosteal vasculature and adjacent musculature, spanning the fracture fragments. It allows micromotion at the fracture site without jeopardize the biology therefore secondary bone healing continue to proceed.

In the current study the final outcome was excellent, good and fair grades according to AOFAS ankle-hindfoot score was regarded as satisfactory group and the poor grade was regarded as unsatisfactory group. So a total of $28(93.34 \%)$ patients were in the satisfactory group and only 2(6.66\%) patients were in unsatisfactory group. In a series of Ahmed Hafiz et al showed in their study that $93.8 \%$ had excellent and good results which was satisfactory and remaining $6.2 \%$ were in poor results which was unsatisfactory ${ }^{13}$. Beside the biologically sound procedure, this technique is easier and faster than the conventional subperiosteal technique in which the lateral periosteum is split to facilitate placement of the plate. We recommend this technique for the treatment of comminuted pronation-abduction type of ankle fracture.

\section{CONCLUSION}

Extraperiosteal plating of pronation-abduction ankle fractures is an effective methods of stabilization that leads to predictable union of the fibular fracture. Pronation-abduction ankle fractures result in laterally comminuted fibular shaft fractures because of the bending moment at the time of fracture. The resultant fragmented bone is challenging to adequately align while maintaining satisfactory vascularity for fracture-healing. A classic direct open fracture reduction and rigid plate fixation will violate the soft-tissue attachments of the fragments and jeopardize the viability of the injured bone.

One way to obtain stability while protecting the soft tissue is to apply a plate outside of the periosteum spanning the fracture fragments, to function as an internal splint. It allows micromotion at the fracture site but does not violate the fracture biology. In the current series, after stabilization of the medial osseous injury (When present) a precontoured plate was placed directly laterally outside of the periosteum, spanning the area of fracture comminution, similar to a bridge plate. The extraperiosteal position avoids the need for bone graft, minimize the risk of infection, and provides the most biologically friendly environment for union allows the soft tissue envelope to be preserved which maintain appropriate length and alignment. All fractures in the present series exhibited a well-aligned mortise and went on to union without bone-grafting. 
In the present series, we found extraperiosteal plating to be an effective method for the stabilization of pronation-abduction ankle fractures. The result of this procedure are at least as good as those of other techniques of open reduction and internal fixation. The technique is easier and faster than standard techniques in which the lateral periosteum is split to facilitate placement of the plate.

\section{DISCLOSURE}

All the authors declared no competing interest.

\section{REFERENCES}

1. Jodi Siegel, Paul Tornetta III. Extraperiosteal Plating of Pronation-Abduction Ankle Fractures. J Bone Joint Surgery.2007(2);89(A):276-281 .

2. Jodi Siegel, Paul Tornetta III. Extraperiosteal Plating of Pronation-Abduction Ankle Fractures. J Bone Joint Surg Am. 2008;90:135-144.

3. T. Schepers. To retain or remove the syndesmotic screw: A review of literature. Arch Orthop Trauma Surg.2011; 131:879-883.

4. Wissing JC, Von Loarhoven CJHM, Vander Werken. The posterior antiglide plate for fixation of fractures of the lateral malleolus.Injury The British journal of accident surgery.1992;23:94-96.

5. Morris N, Lovell M.E.Demographics of 3929 ankle injuries,seasonal variation in diagnosis and more fractures are diagnosed in winter. Injury,Int. J.Care injured. 2012;44:998-1001.

6. Haraguchi N, Robert S. Armiger. A New interpretation of the Mechanism of Ankle Fracture. J Bone Joint Surg Am. 2009;91:821-829.

7. Yih-Shiunn L,Tzu-Liang H,Chien-Rae .Lateral fixation of AO type B ankle fracture: The Acutrak plus compression screw technique. International Orthopaedics (SICOT).2010; 34:903-907.

8. N.Hamid, B.J. Loeffler, W. Braddy, J.F. Kellam, B.E. Cohen,M.J. Bosse. Outcome after fixation of ankle fractures with an injury to the syndesmosis, the effect of the syndesmosis screw. J Bone joint surgery.2009;91B:1069-1073.

9. Sajid E. Rao, Khan AH, Muzammil S. Syndesmosis fixation in bimalleolar webar-c ankle fractures. Professional Med J. 2008;15(1):14-53.

10. Mohammed R, Syed S, Ali SA.Evalution of the syndesmotic only fixation for Weber-C ankle fractures with syndesmotic injury.Indian J Orthop.2011;45(5):454-458.

11. Michel P. J, van den Bekerom, Daniel Haverkamp, Gino M. M. J. Kerkhoffs, C. Niek van Dijk. Syndesmotic Stabilization in Pronation External Rotation Ankle Fractures.Clin Orthop Relat Res.2010;468:991-995.

12. Brad W, Bhandari M, Predictors of functional outcome following trans syndesmotic screw fixation of ankle fractures.J orthop Trauma. 2005;19(2):102-108

13. Ahmad Hafiz Z, Nazri MY,Azril MA, N Premchandran, Ankle fractures: The operative outcome. Malaysian orthopaedic journal. 2011;5(1):40-44. 